\title{
La expresión musical desde el desarrollo auditivo y vocal en Educación Primaria
}

\author{
The musical expression since the auditory and vocal development in primary education
}

\author{
Francisco César Rosa Napal, Isabel Romero Tabeayo \\ Universidade da Coruña, Conservatorio Profesional de Música de A Coruña
}

\begin{abstract}
Resumen
Desde la óptica educativa, la expresión musical puede manifestarse de distintas maneras dependiendo de los objetivos propuestos en cada caso. Por tanto los enfoques sobre la educación musical en la enseñanza general, en los conservatorios y en las facultades universitarias de ciencias de la educación pueden ser contrastados con el fin de generar nuevas líneas de actuación en el proceso enseñanza-aprendizaje de la música. Partiendo de estos principios surgió la iniciativa de realizar una propuesta didáctica de carácter auditivo-vocal dirigida al alumnado de Educación Primaria, en la que participaron docentes de las tres entidades antes mencionadas.
\end{abstract}

Palabras clave: audición, canto, cooperación docente, educación primaria.

\begin{abstract}
From an educational perspective, the musical expression can manifest itself in different ways depending on the objectives proposed in each case. Therefore approaches to music education in general education, in conservatories and university faculties of education can be compared in order to generate new lines of action in the teaching- learning process of music. Starting from these principles emerged the initiative to make a didactic proposal auditory- vocal character aimed at students of primary education, with the participation of teachers from the three aforementioned entities.

Keywords: audition, singing, cooperation teachers, primary education.
\end{abstract}

\section{Fundamentación teórica}

\section{La audición en la educación musical}

Aunque la mayoría de las corrientes pedagógicas de los métodos activos contemplaba en algún momento el aprendizaje de la lectura y la escritura, en línea general se valoró siempre la audición como el primer paso hacia la educación musical.

El aprendizaje de la música y el de la lengua materna se desarrollan en cierto paralelismo atendiendo a las características de cada etapa evolutiva (Willems, 1994). Una de las particularidades de la clasificación que propone el autor es la posición de la escucha en el primer término de su cuadro comparativo.

Por otra parte, Willems (2001) destaca que en correspondencia con los aspectos fisiológicos, afectivos y mentales inherentes al ser humano, es posible entender la audición en esas tres dimensiones:

a. Receptividad sensorial auditiva.

b. Sensibilidad afectivo-auditiva.

c. Percepción mental auditiva.

Estos aspectos, aunque pueden estudiarse de forma aislada, se producen en la práctica de forma simultánea. Con la consideración de éstos se pretende conseguir que el alumnado tenga un contacto directo con los elementos constitutivos de la música antes de manejar la lectoescritura, y es aquí donde esta interpretación de la audición puede justificar en el proceso de aprendizaje de canciones mediante la audición como recurso principal.

\section{La canción en Educación Primaria}

Es necesario destacar que la canción, entendida en toda la amplitud del término, es la forma de expresión en la que predomina el empleo musical de la voz (Randel, 2006). Su presencia en todas las culturas y en todas las épocas se explica por el doble mensaje que encierra al relacionar música y texto en un único modo de expresión.

De todos los contenidos que conforman el área de educación musical posiblemente sea la expresión vocal, a través de la canción, el que tenga el mayor peso. Tradicionalmente, y aún en la actualidad, la canción ha sido empleada no sólo en la enseñanza específica de la música, sino también como un potente recurso didáctico en otras materias, por lo que "en la escuela, el canto es la actividad más global de cuantas se realizan en el ámbito de la educación musical” (Camara, 2004, p. 77).

Partiendo de la propiedad de la canción referente a la combinación en un mismo instante de los dos lenguajes que la constituyen se valoró desde un primer momento su protagonismo en el diseño del proyecto.

\section{Justificación}

La relevancia que podría presentar esta experiencia desde el punto de vista investigativo es que, una vez evaluados los resultados finales y los que se produjeron en el transcurso de las actividades, sería posible concretar un punto partida para la toma de decisiones metodológicas innovadoras por una parte, y por otra, podría generar temas de investigación en ese sentido implicando a un profesorado heterogéneo.

Las líneas de trabajo que podrían derivarse desde el proyecto inicial están dirigidas a dos direcciones fundamentales (Figura1): la primera es encontrar, mediante la suma de experiencias docentes de diferente procedencia, nuevas estrategias para lograr la 
musicalización del alumnado de Primaria de una manera efectiva, motivadora y enriquecedora, mientras la segunda línea es la extrapolación de las conclusiones del trabajo colectivo al ámbito institucional de cada uno de los docentes implicados.

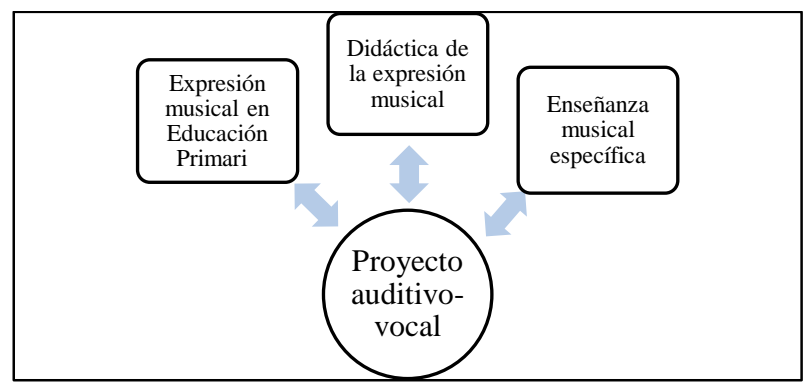

Figura 1. Doble dirección de la experiencia

\section{Objetivos}

En cuanto al trabajo colaborativo. Con esta propuesta didáctica se pretendió unificar criterios educativos y estrategias didácticas a partir de las especificidades profesionales de los docentes participantes. Relacionado con esto, otro objetivo fue el de crear una base que permitiera la continuidad del trabajo de forma sistemática tanto desde la óptica didáctica como desde la investigativa.

En cuanto al propósito didáctico. En este sentido el objetivo fundamental del trabajo fue motivar a todo el alumnado de primaria del centro en la expresión musical desde una perspectiva práctica; aunque también, de forma específica, se pretendía fomentar el trabajo en equipo; desarrollar la comprensión auditiva del alumnado; mejorar la expresión vocal en cuanto a la entonación y la articulación, así como incrementar el interés por el conocimiento de la música popular regional.

\section{Concreción temática}

Partiendo de una proposición de la dirección del centro donde se realizaría el proyecto se acordó enmarcar éste en la temática del día de las letras gallegas, ya que anualmente se realiza un acto en el que se presentan actividades artísticas relacionadas con la fecha, y donde se refleja el trabajo que se ha realizado en el centro. Según este convenio se tomó como eje principal y unificador el empleo de canciones autóctonas de la región, lo que constituyó el principal material didáctico de la experiencia.

\section{Metodología \\ Fase I: Obtención del material}

La tarea de recopilar las piezas requirió una considerable dedicación temporal debido a la gran cantidad de ejemplos existentes, partiendo de que las "melodías y canciones tradicionales, populares o folklóricas, clásicas, modernas y didácticas constituyen el material melódico que se ha de trabajar en el aula" (Carvajo, 1994, p. 13). Debido a la temática elegida, la búsqueda se enfocó hacia el repertorio tradicional gallego en primer lugar, aunque se incursionó en fuentes documentales que recogen canciones compuestas con fines didácticos. También se tuvo en cuenta aquella música que se consiguió en formatos de audio o vídeo.

Se tuvo especial cuidado en la calidad musical y literaria de las canciones, sin embargo se optó por la flexibilidad en los criterios de la coincidencia de las acentuaciones entre el texto y la música, ya que en gran parte del material conseguido constituye un rasgo común la no contemplación de esta práctica.

\section{Fase II: Análisis de los parámetros significativos}

En esta fase se procedió al análisis del material obtenido, para lo que se emplearon los criterios estandarizados de selección. Se tuvo en cuenta aquellos elementos que conforman la melodía, desde las relaciones de la altura, la complejidad de los patrones rítmicos, la extensión y la estructura formal. Siguiendo los criterios que propone Pascual (2002), las melodías deben estar construidas a partir de una interválica congruente con las posibilidades vocales de cada nivel, por lo que se evitó emplear canciones que contuvieran intervalos aumentados o disminuidos o que presentaran una amplitud de más de una quinta justa.

Al igual que la interválica se tuvo también en cuenta el ámbito de las melodías, de manera que en las canciones propuestas para primer ciclo no se superara una sexta, mientras que para segundo y tercer ciclo se estableció como máximo un ámbito de octava justa. En cuanto al ritmo, los parámetros a considerar fueron los relacionados con la métrica. Siguiendo a Pascual (2002), se eligieron las canciones que tuvieran compases binarios de subdivisión binaria para primer y segundo ciclo, pudiendo considerarse viable las subdivisiones ternarias, que abundan en el folklore gallego, en las canciones dirigidas a tercer ciclo. Las melodías con comienzo tético fueron aplicadas a primer ciclo, mientras las que presentaban comienzo anacrúsico pudieron ser destinadas al segundo y al tercero. Al no emplear metodológicamente la lectoescritura convencional, las síncopas y las figuraciones con puntillo, que podrían suponer dificultades en ese contexto no fueron consideradas como inconvenientes, ya que aparecen fundidas con el texto de forma natural.

En todos los casos se veló porque todas las canciones presentaran la simetría formal adecuada al repertorio del proyecto, por lo que siguiendo las recomendaciones de Pascual (2002) se seleccionaron aquellas canciones que presentaban frases de cuatro u ocho compases.

En relación al análisis macro formal se optó por la estructura binaria simple. Por otra parte se atendió a la adecuación de los textos en cuanto a obtener la máxima comprensión en cada curso atendiendo a la complejidad literaria de cada ejemplo.

\section{Fase III: Actividades auditivo-vocales}

Debido a las características y a los objetivos del proyecto se optó por emplear una metodología que garantizara un rápido aprendizaje de las canciones, pero que al mismo tiempo permanecieran en la memoria del alumnado a corto y a largo plazo. Para encontrar la vía óptima de cómo canalizar el proceso de enseñanza y el 
aprendizaje de la música se tuvieron en cuenta las principales modalidades que predominan en el área.

La enseñanza de la expresión musical ha tenido una línea tradicional que se ha extendido en el tiempo y se ha recurrido a ella como único recurso, independientemente de los objetivos que se persiguen en los diferentes niveles y en las diversas vertientes en que se manifiesta.

En este sentido Jorquera (2010) establece los siguientes modelos de enseñanza musical:

a. Modelo académico.

b. Modelo práctico.

c. Modelo comunicativo lúdico.

d. Modelo complejo.

Teniendo en cuenta que cada uno de estos modelos persigue propósitos específicos, se decidió enmarcar el trabajo en dos de ellos de forma complementaria, excluyendo aquellas características propias de cada uno que no se ajustaran a la naturaleza del trabajo. Se eligió el modelo práctico considerando que se desarrolla a través del canto o la interpretación instrumental y está basado en la imitación, que fue la técnica empleada en la enseñanza de las canciones. Por otra parte se empleó el modelo comunicativo lúdico debido a su idoneidad para las tareas propuestas. "La concepción comunicativa lúdica de la enseñanza musical se caracteriza por situar en primer plano la motivación del estudiantado, haciendo que las propuestas del profesor gusten, consiguiendo entretener con actividades amenas" (Jorquera, 2010, p. 7).

Una vez obtenido el material definitivo, y concretados los lineamientos metodológicos, se asignó a cada ciclo dos canciones y se produjo el primer acercamiento a través de la audición activa en cada uno de los grupos, lo que generó el aprendizaje de las canciones de manera casi paralela a la audición.

Los primeros ejercicios realizados estuvieron dirigidos a la respiración atendiendo a que "saber cantar es en gran medida saber respirar. Cuando la respiración es deficiente es necesario implementar las técnicas que permitan un aprovechamiento máximo de la capacidad pulmonar” (Lopardo, 2011, p.100). Al comienzo de la primera sesión se explicó brevemente la técnica de respiración abdominal o diafragmática. Teniendo en cuenta que nunca antes se habían realizado ejercicios de respiración, en las posteriores sesiones encabezarían el conjunto de ejercicios con el fin de lograr cada vez más la recepción de aire. Para evaluar este contenido se realizó un concurso-juego en el que se cronometró el tiempo de inspiración y expiración controlada de forma individual.

Concluidos los ejercicios respiratorios, y continuando esa primera sesión, se procedió a la aplicación de los siguientes pasos en el proceso de aprendizaje de cada una de las canciones:

a. Audición del ejemplo interpretado por los docentes.

b. Imitación colectiva por parte del alumnado cada una de las frases.

c. Audición e interpretación individual de algunos fragmentos en diálogo imitativo.

d. Interpretación colectiva por secciones incorporando los movimientos corporales.

e. Interpretación colectiva de la canción completa a cappella y luego acompañada.
Terminada la actividad auditivo-vocal, se procedió a la grabación en vídeo de las canciones aprendidas que fueron interpretadas por el alumnado y acompañadas instrumentalmente por los docentes para ser empleada más tarde como referencia auditiva y visual en posteriores ensayos realizados bajo la dirección de la profesora del centro.

\section{Fase IV: Evaluación}

La evaluación del trabajo se efectuó al margen de la correspondiente a la asignatura en su carácter oficial, pero se tomaron en consideración aquellos parámetros que forman parte de esta fase.

En este ámbito, Chacón (2012) plantea tres cuestiones básicas que podrían aclarar el proceso de evaluación en el caso específico de la educación musical: ¿Qué significa evaluar en música? ¿Para qué se evalúa en música? ¿Qué otras posiciones existen alrededor del tema?

Contestando a la primera pregunta, se pueden observar cuatro pasos que abarcan todo el contenido evaluable en la educación musical (Chacón, 2012, p. 4):

a. Evaluación (observación de desempeño).

b. Registro (resguardo de información para posterior análisis)

c. Reporte (retroalimentación al estudiante)

d. Acción (cambio, mejoramiento).

En cuanto al propósito de la evaluación en expresión musical el "objetivo primordial de la evaluación en música es generar un cambio tendiente al mejoramiento" (Chacón 2012, p. 4). Este cambio está enfocado en primer lugar al alumnado, aunque también atañe al profesorado, a la familia y a las instituciones.

La tercera pregunta se refiere al consenso que puede surgir al confluir las diferentes concepciones de los demás docentes sobre el papel y el objetivo de la evaluación. En este punto es posible encontrar desde posiciones afines hasta otras totalmente contrastantes (Chacón, 2012).

En el caso específico del trabajo desarrollado, independientemente de la evaluación realizada a partir de las anotaciones, las grabaciones y los ensayos, se empleó también como herramienta el concierto público, en el que fueron interpretadas todas las piezas trabajadas. Aunque la presentación final no constituyó el principal instrumento de evaluación, éste se hizo imprescindible como modo de comprobar los resultados en el momento del análisis comparativo respecto al punto de partida.

\section{Valoración de los resultados}

El resultado del trabajo pudo valorarse positivamente en cada una de sus fases, pues tanto en las sesiones preparatorias, en las que se puso de manifiesto la voluntad y la implicación del profesorado participante, como en las prácticas iniciales, intermedias o en el concierto final donde el alumnado se mostró, en su gran mayoría, entusiasmado con el proyecto.

Uno de los resultados más significativos, que incluso superó las expectativas de los docentes, fue la rapidez con que fueron memorizadas las canciones ya en las primeras actividades auditivo-vocales. Otro de los resultados favorables fue la constatación del 
conocimiento y la práctica de las canciones en contextos diferentes al centro de forma espontánea en actividades externas.

Los problemas que emergieron durante todo el proceso pudieron recogerse de la siguiente manera (Figura 2).

\begin{tabular}{|c|c|}
\hline Parámetros evaluables & Descripción del problema \\
\hline Entonación & $\begin{array}{l}\text { Dependiendo de su edad, una } \\
\text { parte minoritaria del alumnado } \\
\text { muestra mayor o menor temor a } \\
\text { manifestarse mediante la } \\
\text { canción. } \\
\text { Aunque generalmente se logró } \\
\text { una entonación homogénea y } \\
\text { una afinación aceptable, se } \\
\text { detectaron algunos casos } \\
\text { aislados que presentaban } \\
\text { dificultades significativas en } \\
\text { este aspecto. }\end{array}$ \\
\hline Precisión métrica & $\begin{array}{l}\text { La principal dificultad se detectó } \\
\text { en la aplicación de la percusión } \\
\text { corporal simultánea al canto. }\end{array}$ \\
\hline Articulación & $\begin{array}{l}\text { En este aspecto la falta de } \\
\text { entrenamiento en las técnicas de } \\
\text { respiración, y las características } \\
\text { individuales del alumnado } \\
\text { causaron algunas dificultades en } \\
\text { el propósito de conseguir una } \\
\text { articulación equilibrada. }\end{array}$ \\
\hline Expresión & $\begin{array}{l}\text { En algunos momentos de las } \\
\text { canciones fue necesario acudir a } \\
\text { gestos que animaran a expresar } \\
\text { debidamente algunos } \\
\text { fragmentos de canciones, sin } \\
\text { embargo, en determinadas } \\
\text { ocasiones el exceso de } \\
\text { expresividad provocaba la falta } \\
\text { de entonación. }\end{array}$ \\
\hline
\end{tabular}

Figura 2. Dificultades encontradas en el proceso.

\section{Conclusiones}

Entre los propósitos principales de la experiencia realizada, la creación de una plataforma de trabajo colaborativo entre docentes de diferentes centros formativos se pudo establecer como un compromiso estable de actuación anual. En este sentido, surgieron nuevas propuestas en relación con la participación de docentes de otras áreas del conocimiento. De esta manera podrían diseñarse en el futuro trabajos por proyecto más ambiciosos.

En cuanto a la organización se resolvió, por una parte, adelantar la fecha de comienzo de los trabajos preparatorios con el fin de contar con un recorrido temporal más amplio para el trabajo en clase. En otro sentido se generó la necesidad de que cada docente participante elaborara un informe en el que se recogiera, de forma individual, el conjunto de datos obtenidos para ser posteriormente analizados.

Por otra parte, atendiendo a los objetivos relacionados con la propuesta didáctica, se logró de forma global la implicación del alumnado, que al acercarse a la práctica vocal directamente no encontró obstáculos. Al tratarse de actividades de canto colectivo, el propósito de fomentar el trabajo en equipo se vio satisfecho en su totalidad.

Tomando como referencia las dificultades encontradas en todo el proceso, así como las metas conseguidas, es posible concluir que el trabajo desarrollado puede servir como punto de partida para futuras intervenciones didácticas y trabajos de investigación, al mismo tiempo que puede fomentar el trabajo colectivo del profesorado de diferentes entidades educativas y formativas con el fin de crear proyectos interdisciplinares.

\section{Referencias}

Camara, A. (2004). La actividad de cantar en la escuela: una práctica en desuso. Revista Psicodidáctica, № 17, pp. 75-84.

Carvajo, C. (1994). La tarea de enseñar melodías o canciones. Aula de innovación educativa, $\mathrm{N}^{\circ} 24$, pp. 13-16.

Chacón, L. (2012). Qué significa evaluar en música. Revista Electrónica Complutense de Investigación en Educación Musical, Volumen 9, pp 1-25.

Díaz, M. y Giráldez, A. (Coords.) (2007). Aportaciones teóricas y metodológicas a la educación musical. Una selección de autores relevantes. Barcelona: Graó.

Jorquera, M. (2010). Modelos didácticos en la enseñanza musical: el caso de la escuela española. Revista Musical Chilena, $\mathrm{N}^{\circ}$ 214, pp. 52-74.

Lopardo, C. (2011). La entonación en niños de 9 y 10 años: un estudio multicasos. Revista da $A B E M, \mathrm{~N}^{\circ} .25$, pp. 98-112.

Pascual, P. (ed.) (2002). Didáctica de la Música para Primaria. Madrid: Pearson Educación.

Randel, D. (ed.) (2006). Diccionario Harvard de la Música. Madrid. Alianza

Willems, E. (1994). El valor humano de la educación musical. Barcelona: Paidós.

Willems, E. (2001). El oído musical. La preparación auditiva del niño. Barcelona: Paidós. 